PROCEEDINGS OF THE

AMERICAN MATHEMATICAL SOCIETY

Volume 134, Number 2, Pages 591-597

S 0002-9939(05)07965-7

Article electronically published on June 14, 2005

\title{
MONOTONICALLY COUNTABLY PARACOMPACT, COLLECTIONWISE HAUSDORFF SPACES AND MEASURABLE CARDINALS
}

\author{
CHRIS GOOD AND ROBIN W. KNIGHT
}

(Communicated by Alan Dow)

\begin{abstract}
We show that, if an MCP (monotonically countably paracompact) space fails to be collectionwise Hausdorff, then there is a measurable cardinal and that, if there are two measurable cardinals, then there is an MCP space that fails to be collectionwise Hausdorff.
\end{abstract}

A space $X$ is monotonically countably metacompact (MCM) if there is an operator $U$ assigning an open set $U(n, D)$ to each natural number $n \in \omega$ and each closed set $D$ such that:

(1) $D \subseteq U(n, D)$,

(2) if $E \subseteq D$, then $U(n, E) \subseteq U(n, D)$ and

(3) if $\left(D_{n}\right)$ is a decreasing sequence of closed sets with empty intersection, then $\bigcap U\left(n, D_{n}\right)=\varnothing$.

If, in addition, $U$ satisfies

$\left(3^{\prime}\right)$ if $\left(D_{n}\right)$ is a decreasing sequence of closed sets with empty intersection, then $\bigcap \overline{U\left(n, D_{n}\right)}=\varnothing$

then $X$ is said to be monotonically countably paracompact or MCP. MCP spaces were introduced by the authors and Stares [3], and this (somewhat simplified) definition was shown to be equivalent by Ge and the first author [5], where it was also shown that one can assume that $U(n, D)=\bigcup_{x \in D} U(n, x)$ (writing $U(n, x)$ for $U(n,\{x\}))$. Note also that, if $U^{\prime}(n, x) \subseteq U(n, x)$ for all $n \in \omega$ and $x \in X$, then $U^{\prime}(n, D)=\bigcup_{x \in D} U^{\prime}(n, x)$ also defines an MCP operator.

Without the monotonicity condition (2), conditions (1) and $\left(3^{\prime}\right)$ characterize countable paracompactness, a covering property which plays an important rôle in the preservation of normality in products 12, 13. However, MCM and MCP are really generalized metric properties: MCM is equivalent to Hodel's notion of a $\beta$-space and MCP is a generalization of stratifiability, rather than a monotonized covering property (see [3, 5] for details and definitions).

It turns out that a number of set theoretic results concerning the separation of closed discrete collections in normal spaces have direct analogues for countably paracompact spaces. For example, Burke [1] extends Nyikos's elegant 'provisional'

Received by the editors July 30, 2003 and, in revised form, September 9, 2004.

2000 Mathematics Subject Classification. Primary 54C10, 54D15, 54D20, 54E20, 54E30.

Key words and phrases. Monotone countable paracompactness, MCP, collectionwise Hausdorff, measurable cardinals.

(C)2005 American Mathematical Society Reverts to public domain 28 years from publication 
solution to the normal Moore space problem by showing that countably paracompact, Moore spaces are metrizable assuming PMEA, and Watson [14] shows that, assuming $V=L$, first countable, countably paracompact spaces are collectionwise Hausdorff.

When normality is replaced by monotone normality (see 8 for a definition), settheoretic assumptions are usually not needed for these theorems, and it turns out [3] that the same is often true if countable paracompactness is replaced by MCP. For example, every MCP Moore space is metrizable and every first countable or locally compact MCP space is collectionwise Hausdorff. Of course, every monotonically normal space is collectionwise normal and hence collectionwise Hausdorff, so it is natural to ask whether every MCP space is collectionwise Hausdorff. (A space is collectionwise Hausdorff provided every closed discrete subset can be separated by disjoint open sets, i.e. for each $x \in D$ there is an open set $U_{x}$ containing $x$ such that $U_{x} \cap U_{y}=\varnothing$ whenever $x \neq y \in D$.)

The answer to this question is somewhat intriguing. Whereas results about monotonic properties rarely involve set-theoretic assumptions, we prove here that if there is an MCP space which fails to be collectionwise Hausdorff, then there is a measurable cardinal, whilst if there are two measurable cardinals, there is an MCP space that is not collectionwise Hausdorff. We also observe that PMEA does not imply that MCP spaces are collectionwise Hausdorff.

Our notation and terminology are standard as found in, say [6, 10. We denote the set of all natural numbers by $\omega$ and the power set of a set $A$ by $\mathcal{P}(A)$. All spaces are regular and $T_{1}$. An uncountable cardinal $\kappa$ is measurable if there exists a $\kappa$-complete free ultrafilter on $\kappa$ (i.e. an ultrafilter, which has empty intersection, with the property that the intersection of fewer than $\kappa$ many elements of the filter is again in the filter). The existence of any countably complete free ultrafilter on any set implies the existence of an uncountable measurable cardinal.

Theorem 1. If there is a regular MCP space that is not collectionwise Hausdorff, then there is a measurable cardinal.

Proof. Let $X$ be a regular MCP space and $D$ a closed discrete subset of $X$ that cannot be separated by disjoint open sets. Let $V$ be an MCP operator such that $V(n, C)=\bigcup_{x \in C} V(n, x)$, for every closed subset $C$ of $X$, and (by regularity) $\overline{V(n+1, x)} \subseteq V(n, x)$, for each $x \in X$.

Claim 1. There is a finer regular topology on $X$ with MCP operator $U$ with respect to which:

(1) $D$ is closed discrete and cannot be separated by disjoint open sets;

(2) each $x \in X \backslash D$ is an isolated point with $U(n, x)=\{x\}$ for all $n \in \omega$;

(3) for each $x \in D, \overline{U(1, x)} \cap D=\{x\}$ and $\overline{U(n+1, x)} \subseteq U(n, x)$;

(4) $U(n, C)=\bigcup_{x \in C} U(n, x)$, for each closed $C \subseteq X$;

(5) $\overline{U(n, A)} \cup \overline{U(n, B \backslash A)}=\overline{U(n, B)}$, whenever $A \subseteq B \subseteq D$.

Proof of Claim 1. Refine the topology on $X$ by adding the collection $\{\{x\}: x \notin D\}$ of new open sets: it is easy to see that the resulting space is again regular, MCP but not collectionwise Hausdorff.

Define $U(n, x)=\{x\}$ for each $x \notin D$. Since $X$ is regular and $D$ is closed discrete, for each $x \in D$ there is an open $V_{x}$ such that $\overline{V_{x}} \cap D=\{x\}$. Define 


$$
\begin{aligned}
& U(n, x)=V(n, x) \cap V_{x} . \text { Note that whenever } A \subseteq B \subseteq D \\
& \overline{U(n, A)} \cup \overline{U(n, B \backslash A)}=\overline{U(n, A) \cup U(n, B \backslash A)}=\overline{U(n, B) .}
\end{aligned}
$$

The other conditions are easy to verify.

Now, for each $x \in D$, define

$$
U_{x}=\bigcup_{n \in \omega}[U(n, x) \backslash \overline{U(n, D \backslash\{x\})}] .
$$

Claim 2. The collection $\left\{U_{x}: x \in D\right\}$ is a pairwise disjoint collection of open sets. Moreover, there is some $x \in D$ such that $x \in \bigcap_{n \in \omega} \overline{U(n, D \backslash\{x\})}$.

Proof of Claim 2. By monotonicity, if $y \neq x$, then $U(n, y)$ is a subset of the open set $U(n, D \backslash\{x\})$ for each $n \in \omega$, so that $U_{x} \cap U_{y}=\varnothing$ and $\left\{U_{x}: x \in D\right\}$ is a pairwise disjoint collection of open sets. Obviously, as $D$ cannot be separated by open sets, there must be some $x \in D$ such that $x \notin U_{x}$ so that $x \in \bigcap_{n \in \omega} \overline{U(n, D \backslash\{x\})}$.

Fix such an $x$ and consider the collection

$$
\mathcal{D}_{x}=\left\{E \subseteq D \backslash\{x\}: x \in \bigcap_{n \in \omega} \overline{U(n, E)}\right\} .
$$

By the above comments, to show that there is a measurable cardinal, it is enough to find a subset of $\mathcal{D}_{x}$ that forms a countably complete free ultrafilter on some subset of $D$. Certainly, since $x$ is not in $U_{x},(D \backslash\{x\}) \in \mathcal{D}_{x} \neq \varnothing$, but $\mathcal{D}_{x}$ itself need not be a filter since, quite possibly, it contains disjoint subsets.

Claim 3. If $\mathcal{D}$ a collection of subsets of $D$ such that $\bigcap \overline{U\left(n, G_{n}\right)} \neq \varnothing$, whenever $\left\{G_{n}: n \in \omega\right\} \subseteq \mathcal{D}$, then there is some $F \in \mathcal{D}$ such that $G \cap H \neq \varnothing$ whenever $G, H \in \mathcal{D}$ and $G \cup H \subseteq F$.

Proof of Claim 3. We build a tree $T$ of elements of $\mathcal{D}$ with root $D$ and levels $T_{n}$, $n \in \omega$, as follows. Given $E \in T_{n}$, choose if possible some disjoint $F_{E}, G_{E} \subseteq E$ such that both $F_{E}$ and $G_{E}$ are in $\mathcal{D}$ and let the successors of $E$ be $F_{E}$ and $G_{E}$. If no such $F$ exists, $E$ has no successors. $T$ is a finitely (two) branching countable tree and, by König's Lemma, if it is infinite, then it has an infinite branch, $D=$ $E_{0}<E_{1}<E_{2}<\cdots$ such that (re-labelling as necessary) $E_{n+1}=F_{E_{n}}$. Note that $E_{n}<G_{E_{n}} \in \mathcal{D}$ and $\left\{G_{E_{n}}: n \in \omega\right\}$ is an infinite, pairwise disjoint, subcollection of $\mathcal{D}$. But now $\left\{D_{n}=\bigcup_{j>n} G_{E_{j}}: n \in \omega\right\}$ is a decreasing sequence of closed subsets of $X$ with empty intersection, which is impossible, since $U$ is an MCP operator and yet

$$
\varnothing \neq \bigcap_{n \in \omega} \overline{U\left(n, G_{n}\right)} \subseteq \bigcap_{n \in \omega} \overline{U\left(n, D_{n}\right)} .
$$

Hence $T$ is finite and has some maximal element $F \in \mathcal{D}$.

As $x \in \overline{U(n, E)}$ for all $E \in \mathcal{D}_{x}$, Claim 3 implies that there is some $F \subseteq D$ such that $G \cap H \neq \varnothing$ whenever $G, H \in \mathcal{D}_{x}$ and $G \cup H \subseteq F$. It remains to show that

$$
\mathcal{F}=\left\{E \subseteq F: x \in \bigcap_{n \in \omega} \overline{U(n, E)}\right\}
$$

is a countably complete free ultrafilter. 
Since $F \in \mathcal{F}, \mathcal{F} \neq \varnothing$ and clearly $G$ is in $\mathcal{F}$ whenever $G \supseteq H \in \mathcal{F}$. Suppose that $G$ and $H$ are in $\mathcal{F}$. Since $x \in \bigcap_{n \in \omega} \overline{U(n, G)}, x \in \overline{U(n, G)}=\overline{\overline{U(n, G \cap H)}} \cup \overline{U(n, G \backslash H)}$ and $U(n+1, A) \subseteq U(n, A)$ for any $A \subseteq D, x$ is in either $G_{0}=\bigcap_{n \in \omega} \overline{U(n, G \cap H)}$ or $G_{1}=\bigcap_{n \in \omega} \overline{U(n, G \backslash H)}$. If $G \cap H$ is not in $\mathcal{F}$, then $x$ is not in $G_{0}$ but is in $G_{1}$. This implies that both $G \backslash H$ and $H$ are both in $\mathcal{F}$, which contradicts the definition of $F$. Moreover, if $G \in \mathcal{F}$ and $K \subseteq G$, then

$$
x \in \bigcap_{n \in \omega} \overline{U(n, G)} \subseteq \bigcap_{n \in \omega} \overline{U(n, K)} \cup \bigcap_{n \in \omega} \overline{U(n, G \backslash K)}
$$

so either $K$ or $G \backslash K$ is in $\mathcal{F}$. Hence $\mathcal{F}$ is an ultrafilter.

To see that $\mathcal{F}$ is free note that $x \notin \bigcap_{n \in \omega} \overline{U(n,\{y\})}$ for any $y \in F$, so that $G \backslash\{y\} \in \mathcal{F}$, for any $G \in \mathcal{F}$, and $\bigcap \mathcal{F}=\varnothing$.

Finally, to see that $\mathcal{F}$ is countably complete, suppose that $G_{n} \in \mathcal{F}$ and $G=$ $\bigcap_{n \in \omega} G_{n}$. If $\bigcap_{n \in \omega} G_{n}=\varnothing$, then $\bigcap \overline{U\left(n, G_{n}\right)}=\varnothing$, which is impossible since $x \in \overline{U\left(n, G_{n}\right)}$ for each $n$. But then $G$ must be in $\mathcal{F}$, since otherwise $G_{n} \backslash G \in \mathcal{F}$ and certainly $\bigcap_{n \in \omega}\left(G_{n} \backslash G\right)=\varnothing$.

As a partial converse to Theorem 1 we have the following example.

Example 2. If there are two measurable cardinals, then there is a regular MCP space that is not collectionwise Hausdorff.

Proof. We use an idea from [4]. Let $\kappa<\lambda$ be two measurable cardinals, $\mathcal{U}$ a $\kappa$ complete ultrafilter on $\kappa$ and $\mathcal{V}$ a $\lambda$-complete ultrafilter on $\lambda$. Defining $S^{\alpha}=\{\beta \in$ $\lambda:(\alpha, \beta) \in S\}$ and $S_{\beta}=\{\alpha \in \kappa:(\alpha, \beta) \in S\}$ for each $S \subseteq \kappa \times \lambda$, we have filters

$$
\begin{aligned}
& \mathcal{U} \cdot \mathcal{V}=\left\{S \subseteq \kappa \times \lambda:\left\{\alpha \in \kappa: S^{\alpha} \in \mathcal{V}\right\} \in \mathcal{U}\right\}, \\
& \mathcal{U} * \mathcal{V}=\left\{S \subseteq \kappa \times \lambda:\left\{\beta \in \lambda: S_{\beta} \in \mathcal{U}\right\} \in \mathcal{V}\right\},
\end{aligned}
$$

on $\kappa \times \lambda$. Moreover, since $\mathcal{U}$ is $\kappa$-complete and $\mathcal{V}$ is $\lambda$-complete, $\mathcal{U} \cdot \mathcal{V}=\mathcal{U} * \mathcal{V}$ is a $\kappa$-complete free ultrafilter. For details we refer the reader to [2, 4].

Let the set $K=\kappa+1=\{\alpha: \alpha \leq \kappa\}$ have the topology generated by isolating each $\alpha \in \kappa$ and declaring open sets of the point $\kappa$ to be of the form $\{\kappa\} \cup U$ for any $U \in \mathcal{U}$. Similarly, topologize $L=\lambda+1$ by declaring each $\alpha \in \lambda$ to be isolated and open neighbourhoods of $\lambda$ to have the form $\{\lambda\} \cup V$ for some $V \in \mathcal{V}$. Let $K \times L$ have the usual product topology and let $X$ be the subspace $(K \times L) \backslash\{(\kappa, \lambda)\}$. Let $K_{0}=\{(\alpha, \lambda): \alpha \in \kappa\}$ and $L_{0}=\{(\kappa, \alpha): \alpha \in \lambda\}$.

Since $K$ and $L$ are both regular, $X$ is regular. The set $K_{0} \cup L_{0}$ is clearly a closed discrete subset of $X$ and, if $X$ were collectionwise Hausdorff, then the two disjoint closed sets $K_{0}$ and $L_{0}$ would have disjoint open neighbourhoods $U_{K}$ and $U_{L}$. Clearly $U_{K} \in \mathcal{U} \cdot \mathcal{V}$ and $U_{L} \in \mathcal{U} * \mathcal{V}$, but this is a contradiction as $\mathcal{U} \cdot \mathcal{V}=\mathcal{U} * \mathcal{V}$, so that $U_{K} \cap U_{L} \neq \varnothing$.

To see that $X$ is MCP, note first that it suffices to consider only decreasing closed subsets of $K_{0} \cup L_{0}$, since all other points are isolated. For each $n \in \omega$ and each $\alpha \in \kappa$ let $U(n,(\alpha, \lambda))=\{\alpha\} \times L$ and for each $\beta \in \lambda$ let $U(n,(\kappa, \beta))=K \times\{\beta\}$. If $\left\{D_{n}\right\}$ is a decreasing sequence of (closed) subsets of $K_{0} \cup L_{0}$ there must be some $N$ such that for all $n \geq N$ neither $\left\{\alpha:(\alpha, \lambda) \in D_{n}\right\} \in \mathcal{U}$ nor $\left\{\beta:(\kappa, \beta) \in D_{n}\right\} \in \mathcal{V}$. 
But then, for $n \geq N$,

$$
\begin{aligned}
\overline{U\left(n, D_{n}\right)} & =\overline{\bigcup_{(\alpha, \lambda) \in D_{n}} U(n,(\alpha, \lambda)) \cup \bigcup_{(\kappa, \beta) \in D_{n}} U(n,(\kappa, \beta))} \\
& =\bigcup_{\left\{\alpha:(\alpha, \lambda) \in D_{n}\right\}}\{\alpha\} \times L \cup \frac{\bigcup_{\left\{\beta:(\kappa, \beta) \in D_{n}\right\}} K \times\{\beta\}}{\bigcup_{\left\{\alpha:(\alpha, \lambda) \in D_{n}\right\}}\{\alpha\} \times L \cup \underset{\left.\{\kappa, \beta) \in D_{n}\right\}}{ } K \times\{\beta\} .}
\end{aligned}
$$

Hence $\bigcap_{n \in \omega} \overline{U\left(n, D_{n}\right)}=\varnothing$.

So is the existence of an MCP space that is not collectionwise Hausdorff equivalent to the existence of one or of two measurables? This appears to be a hard question, as the next two results suggest. Theorem 3 says that in any case the cardinality of the closed discrete, unseparated set in any example must be greater than the first measurable, whilst Proposition 4 implies that the structure of the space described in Example 2 is, without loss of generality, canonical. The construction of the example really requires the existence of two distinct countably complete ultrafilters $\mathcal{U}$ and $\mathcal{V}$ such that $\mathcal{U} \cdot \mathcal{V}=\mathcal{U} * \mathcal{V}$. We do not know whether this follows from the existence of a single measurable.

Theorem 3. Let $\kappa$ be the first measurable cardinal and suppose that $X$ is a regular $M C P$ space that is not collectionwise Hausdorff. If $D$ is a closed discrete subset of $X$ that cannot be separated by open sets, then $\kappa<|D|$.

Proof. Without loss of generality, suppose that $X$ is as in the conclusion of Claim 1 of Theorem 1] with MCP operator $U$.

Suppose that $D$ has cardinality no greater than $\kappa$ and is indexed as $D=\left\{x_{\alpha}\right.$ : $\alpha \in \lambda\}$ for some $\lambda \leq \kappa$. For each $\alpha<\kappa$, let $D^{\alpha}=\left\{x_{\beta}: \beta<\alpha\right\}$ and let $\lambda_{\alpha}$ be the cardinality of $\alpha$. Arguing as in Theorem 1, if $x_{\alpha} \in \bigcap_{n \in \omega} \overline{U\left(n, D^{\alpha}\right)}$, then there is a countably complete free ultrafilter on the set $\alpha$ of cardinality $\lambda_{\alpha}$, contradicting the fact that $\kappa$ is the least measurable. Hence there is some open $V_{\alpha}$ and some $n_{\alpha} \in \omega$ such that $x_{\alpha} \in V_{\alpha}$ and $V_{\alpha} \cap \overline{U\left(n, D^{\alpha}\right)}=\varnothing$ for all $n \geq n_{\alpha}$.

Let $D_{n}=\left\{x_{\alpha}: n_{\alpha} \leq n\right\}$ and, for each $\alpha$, let $W(\alpha, n)=V_{\alpha} \cap U\left(n, x_{\alpha}\right)$. If $x_{\alpha}$ and $x_{\beta}$ are in $D_{n}$, then $n$ is at least as big as both $n_{\alpha}$ and $n_{\beta}$ and without loss of generality $\beta<\alpha$. But then $V_{\alpha} \cap U\left(n_{\alpha}, D^{\alpha}\right)=\varnothing$, which implies by monotonicity that $V_{\alpha} \cap U\left(n, x_{\beta}\right)=\varnothing$. Hence $W(\alpha, n)$ and $W(\beta, n)$ are disjoint and $D_{n}$ is screened by $\left\{W(\alpha, n): x_{\alpha} \in D_{n}\right\}$.

Now let $W$ be the MCP operator defined by $W(n,\{x\})=\{x\}$, for $x \notin D$ and for $x \in D, W\left(n,\left\{x_{\alpha}\right\}\right)=W\left(\alpha, n_{\alpha}\right), n \leq n_{\alpha}$, and $W\left(n,\left\{x_{\alpha}\right\}\right)=W(\alpha, n)$, for each $n \geq n_{\alpha}$. Arguing as in Theorem 1, there is some $x \in D$ such that $x \in \bigcap_{n \in \omega} \overline{W(n, D \backslash\{x\})}$ and some subset $F$ of $D \backslash\{x\}$ on which there is a countably complete ultrafilter $\mathcal{F}=\left\{E \subseteq F: x \in \bigcap_{n \in \omega} \overline{W(n, E)}\right\}$. Since $\left\{W(\alpha, n): x_{\alpha} \in D_{n}\right\}$ separates $D_{n}$, if $x \in D_{n}$,

$$
x \notin \overline{W\left(n, D_{n} \backslash\{x\}\right)}=\overline{\bigcup\left\{W(\alpha, n): x_{\alpha} \in D_{n} \backslash\{x\}\right\}} .
$$

This implies that $D_{n} \cap F$ is not in $\mathcal{F}$, so that $F_{n}=F \backslash D_{n}$ is in $\mathcal{F}$. But $\bigcup_{n \in \omega} D_{n}=D$ so that $\bigcap_{n \in \omega} F_{n}=\varnothing$, contradicting the fact that $\mathcal{F}$ is countably complete. 
Proposition 4. Let $X$ be a regular $M C P$ space, $D$ a closed discrete subset and $A$ a subset of $D$ such that $A$ and $D \backslash A$ cannot be separated by disjoint open sets. Then there is a regular space $Y$ with underlying set $X$ and $M C P$ operator $U$ with respect to which

(1) $U$ and $D$ satisfy conditions (1)-(5) of Claim 1 of Theorem 1,

(2) $A$ and $D \backslash A$ cannot be separated by disjoint open sets,

(3) $\{U(1, x): x \in A\}$ and $\{U(1, x): x \in D \backslash A\}$ are pairwise disjoint collections.

Proof. Again without loss of generality we may assume that $X$ is as in the conclusion of Claim 1 of Theorem 1 and has MCP operator $V$. (The refinement of the topology in Claim 1 does not introduce a separation of the disjoint closed sets $A$ and $D \backslash A$ by disjoint open sets.)

Let $X_{0}=X \backslash D$ and $B=D \backslash A$. Choose distinct points $z_{x, y}$ (not in $X$ ) for each $x \in A, y \in B$ and $z \in V(1, x) \cap V(1, y) \subseteq X_{0}$. Let $Y=D \cup\left\{z_{x, y}: x \in A, y \in B, z \in\right.$ $V(1, x) \cap V(1, y)\}$. We topologize $Y$ as follows. If $z \in Y \backslash D$, then $z$ is isolated. If $x \in A$, then a basic open set about $x$ takes the form

$$
U_{W}=\{x\} \cup\left\{z_{x, y}: z \in W \cap V(1, y) \cap X_{0}, y \in B\right\},
$$

where $W$ is a basic open neighbourhood of $x$ in $X$. Similarly, basic open neighbourhoods of $y \in A$ take the form

$$
U_{W}=\{y\} \cup\left\{z_{x, y}: z \in W \cap V(1, x) \cap X_{0}, x \in A\right\},
$$

where $U$ is an open neighbourhood of $y$ in $X$.

It is easy to check that $Y$ with this topology is $T_{1}$ and 0-dimensional, hence regular. Let $U(n, z)=\{z\}$ for all $z \in Y \backslash D$ and $U(n, x)=U_{V(n, x)}$. It is clear that both $\{U(1, x): x \in A\}$ and $\{U(1, x): x \in B\}$ are pairwise disjoint collections. To check that $U$ defines an MCP operator, it is enough to assume that $\left(D_{n}\right)$ is a decreasing sequence of subsets of $D$ with empty intersection. As $V$ is an MCP operator, $\bigcap_{n \in \omega} \overline{\bigcup_{z \in D_{n}} V(n, z)}=\varnothing$. Suppose that $x \in \bigcap_{n \in \omega} \overline{\bigcup_{z \in D_{n}} U(n, z)}$. Then $x \in D$ and there is some open neighbourhood $W$ of $x$ in $X$ such that $W \cap \bigcup_{z \in D_{n}} V(n, z)=\varnothing$ for all $n$ larger than some $N$, which implies that $U_{W} \cap \bigcup_{z \in D_{n}} U_{V(n, z)}=\varnothing$. Hence $\bigcap_{n \in \omega} \overline{\bigcup_{z \in D_{n}} U(n, z)}=\varnothing$. A similar argument shows that $A$ and $B$ cannot be separated by disjoint open sets in $Y$, which, in turn, implies that the points of $D$ cannot be separated by disjoint open sets.

Finally we note that PMEA does not imply that MCP spaces are collectionwise Hausdorff. Levy and Solovay 11 prove that measurability is preserved in mild Cohen extensions (that is, if $\lambda$ is a measurable cardinal and $\mathbb{P}$ is a forcing poset of $<\lambda$ conditions, then $\lambda$ is measurable in the forcing extension). PMEA holds in any model obtained by adding supercompact many random reals (see [7]). Hence, if $\kappa<\lambda<\mu$, where $\kappa$ is supercompact and $\lambda$ and $\mu$ are measurable, then adding $\kappa$ many Cohen reals results in a model of PMEA containing two measurables and, by Example 2, an MCP space that is not collectionwise Hausdorff.

\section{ACKNOWLEDGEMENT}

We are most grateful to the referee for his comments and for his suggestions for improving the presentation of the paper. 


\section{REFERENCES}

[1] D. Burke, PMEA and first countable, countably paracompact spaces, Proc. Amer. Math. Soc., 92 (1984), 455-460. MR0759673 (85h:54032)

[2] W.W. Comfort, and S. Negrepontis, The theory of ultrafilters (Springer-Verlag, Berlin, 1974). MR0396267 (53:135)

[3] C. Good, R. W. Knight and I. S. Stares, Monotone Countable Paracompactness, Topol. Appl., 101 (2000), 281-298. MR1733809 (2000k:54024)

[4] C. Good, D. W. McIntyre, W. S. Watson, Measurable cardinals and finite intervals between regular topologies, Topol. Appl., 123 (2002), 429-441. MR.1924043(2003h:54001)

[5] C. Good and G. Ying, A note on monotone countable paracompactness, Comment. Math. Univ. Carolinae, 42 (2001), 771-778. MR.1883385 (2003a:54027)

[6] R. Engelking, General Topology, (Heldermann Verlag, Berlin 1989). MR.1039321 (91c:54001)

[7] W. G. Fleissner, The normal Moore space conjecture and large cardinals, in Handbook of settheoretic topology, K. Kunen and J. E. Vaughan, eds. (North-Holland, Amsterdam, 1984). MR0776635 (86m:54023)

[8] G. Gruenhage, Generalized metric spaces, in Handbook of set-theoretic topology, K. Kunen and J. E. Vaughan, eds. (North-Holland, Amsterdam, 1984). MR0776629 (86h:54038)

[9] R. E. Hodel, Spaces defined by sequences of open covers which guarantee that certian sequences have cluster points, Proceedings of the University of Houston Point Set Topology Conference (Houston, Tex., 1971), (1971), 105-114. MR0407810 (53:11580)

[10] K. Kunen, Set Theory, An Introduction to Independence Proofs, North Holland, Amsterdam (1983). MR0756630 (85e:03003)

[11] A. Lévy and R. M. Solovay, Measurable cardinals and the continuum hypothesis, Israel J. Math., 5 (1967), 234-248. MR0224458 (37:57)

[12] T. C. Przymusiński, Products of normal spaces, in Handbook of set-theoretic topology, K. Kunen and J. E. Vaughan, eds. (North-Holland, Amsterdam, 1984). MR0776637 (86c:54007)

[13] M. E. Rudin, Dowker spaces, in Handbook of set-theoretic topology, K. Kunen and J. E. Vaughan, eds. (North-Holland, Amsterdam, 1984). MR0776636 (86c:54018)

[14] W. S. Watson, Separation in countably paracompact spaces, Trans. Amer. Math. Soc., 290 (1985), 831-842. MR0792831(87b:54016)

School of Mathematics and Statistics, University of Birmingham, Birmingham B15 2TT, United KingDOM

E-mail address: c.good@bham.ac.uk

Mathematical Institute, University of Oxford, 24-29 St Giles', Oxford OX1 3LB, UNITED KingDOM

E-mail address: knight@maths.ox.ac.uk 